\title{
Discovery of novel microorganisms involved in ergot alkaloid detoxification: An approach
}

\section{S. PERUMBAKKAM ${ }^{1}$, R. M. RATTRAY ${ }^{2}$, M. J. M. DELORME 2 , J. M. DURINGER ${ }^{1}$ and A. M. CRAIG} ${ }^{1}$ Department of Environmental and Molecular Toxicology ${ }^{2}$ Department of Microbiology ${ }^{3}$ College of Veterinary Medicine Oregon State University, 139 Oak Creek Building, Corvallis, Oregon 97331, USA a.morrie.craig@oregonstate.edu

\begin{abstract}
Fescue toxicosis and ergot alkaloids are estimated by the United States Department of Agriculture (USDA) to cost the U.S. ranchers $\$ 860$ million per year. The endophyte fungi Neotyphodium coenophialum is present in tall fescue (Festuca arundinacea). This fungus produces toxic alkaloids (principally ergovaline) that cause deleterious effects in cattle and other livestock when infected grasses are eaten. Previous studies in sheep and cattle have yielded no culturable microbes able to detoxify the ergot molecules, leading to the search for novel sources of anaerobic organisms capable of degrading ergot alkaloids. Anaerobic incubation of earthworm (Eisenea fetida) homogenate with pure ergovaline has exhibited a $60 \%$ decrease in ergovaline concentration. Digestive tracks were dissected from worms obtained from an endophyte-fed digester. Cloning and partial sequencing of bacterial $16 \mathrm{~S}$ rDNA obtained from these samples revealed a diverse microbiota, including bacteria sequences which classified into the phyla Bacteroidetes, Firmicutes, Proteobacteria, and Planktomycetes. Future work is focused on refining our understanding of the contribution towards possible ergot alkaloids detoxification made by the bacteria of the worm-gut microbiota.
\end{abstract}

Keywords: ergovaline, microbial degradation, endophyte, tall fescue, perennial ryegrass, Eisenea fetida

\section{Introduction}

Ergotism caused by the consumption of ergot alkaloids has been known for many centuries. Specific chemical composition and concentrations of ergot alkaloids vary with the symbiotic combination between the grass and the fungus. In tall fescue (Festuca arundinacea) infected with Neotyphodium coenophialum ( $N$. coenophialum), the fungus forms a symbiotic relation and produces ergovaline as its predominant ergot alkaloid (Arechavaleta et al. 1992). Neotyphodium lolii (N. lolii) associates with perennial ryegrass (Lolium perenne) and also produces ergovaline.

Tall fescue grows on about 14 million hectares in the United States and is the most widely grown pasture grass in humid areas of the southeastern and to a lesser extent in the northwestern United States (Buckner et al. 1979). Unfortunately, the alkaloids produced by the endophyte cause deleterious effects in cattle and other herbivore species when endophyte-infected grasses are grazed or fed as hay. In mammals, ergot alkaloids affect the central and sympathetic nervous systems, as well as immune and reproductive systems, resulting in symptoms such as muscle contractions, changes in blood pressure, lowered immune response, reduced lactation and reproductive capability, disturbances in sleep/wake cycles, hallucinations, and gangrene of the extremities (Porter \& Thompson Jr. 1992). Ergot alkaloids also affect other organisms including bacteria, nematodes, and insects.
The economic loss to the U.S. beef cattle industry of tall fescue toxicosis is estimated at about $\$ 860$ million. Fescue foot or summer syndrome, ryegrass staggers, and reproductive problems caused by these plant toxins cause $\$ 1$ billion in livestock losses annually in the United States alone (Browning Jr. 2004). Research laboratories across the country are working on different strategies to address the endophyte problem. To date, none have provided the ultimate solution to this problem. Three scientific solutions have been proposed to solve the dilemma of ergovaline. The first involves genetic alteration of endophyte in tall fescue or perennial ryegrass seed to produce ergot alkaloids selective against insects rather than herbivores. Second alternative was to develop a vaccine that would allow the animal to systemically bind the toxic alkaloids. The third alternative would be to isolate anaerobic bacteria that possess ability to degrade ergot alkaloids before systemic absorption. Microorganisms are versatile in mineralising numerous toxic substances.

Previous studies in sheep and cattle have yielded no cultivable microbes able to detoxify ergot molecules, leading to the search for novel sources of anaerobic organisms such as in the gut of "red wiggler" earthworm (Eisenea fetida), capable of degrading ergot alkaloids. We incubated ergovaline spiked media with earthworm gut contents. Further, DNA from the earthworm gut was isolated and a $16 \mathrm{~S}$ rDNA library constructed to identify native bacteria of the earthworm.

\section{Materials and Methods}

\section{Sample collection and media}

Earthworms (Eisenia fetida) were randomly collected from a compost bin which was previously seeded with ergovaline infected seed and grass clippings. Earthworms weighing 21.8 $\mathrm{g}$ were dipped/washed in $50 \%$ ethanol. The surface sterilised earthworms were transferred to an anaerobic glove box (Coy, Grass Lake, MI). In the anaerobic glove box, the earthworms were ground using a tissue homogeniser and $50 \mathrm{~mL}$ of McDougall's buffer (McDougall 1948). One mL of worm slurry was incubated with $10 \mathrm{~mL}$ media $\mathrm{E}$ in Balch tubes. Ergovaline crystals, obtained from Forest Smith (Dept. Pharmacal Sciences, Auburn University, AL), was added to each tube at a concentration of $150 \mathrm{ppb}$. Balch tubes were incubated at $38^{\circ} \mathrm{C}$ and samples were drawn anareobically at 0 h, 2 day, 4, and 7 days using disposable syringes. The samples were then transferred to glass tubes and extraction was carried out by HPLC. All experiments were performed in duplicate.

\section{Isolation and Extraction of DNA from earthworm gut} Two worms were soaked in $50 \%$ ethanol solution for $10 \mathrm{~min}$ before dissection. Using a No.20 scalpel blade the crop/gizzard and anus section of the worm were removed. Then a lateral incision was made to remove the outer covering of worm and to isolate the gut. The worm gut was then placed into a microfuge tube, and sterile phosphate buffered saline (PBS) added to 
homogenise the worm gut. DNA was then isolated from worm gut homogenate using a DNA extraction kit (Mobio, Carlsbad, CA). The final purified DNA was visualised by running samples on a $1 \%$ agarose gel.

\section{PCR amplification and construction of 16S rDNA clone library}

The DNA samples from the worm gut were subjected to PCR amplification using primers fD1 and rP2 (Weisburg et al. 1991). The PCR reaction was carried out using the TripleMaster PCR system (Eppendorf, Hamburg, Germany) in a PTC-200 thermocycler (MJ Research Inc., Watertown, MA). PCR products were purified using the QIAquick PCR purification kit (Qiagen Inc., Valencia, CA). The purified DNA was cloned into the TOPO ${ }^{\circledR}$ 4 vector system (Invitrogen, Carlsbad, CA) and transformation of One Shot ${ }^{\mathbb{E}}$ TOP10 (Invitrogen) chemically competent $E$. coli cells were done according to the manufacturer's protocol. The LB plates were incubated at $37^{\circ} \mathrm{C}$ overnight and transformants picked and transferred to 96 well plates containing $1.6 \mathrm{~mL}$ of $2 \mathrm{x}$ LB broth amended with $100 \mu \mathrm{g} \mathrm{mL}^{-1}$ of ampicillin and shaken (250 rpm) at $37^{\circ} \mathrm{C}$ overnight. A Montage Plasmid Miniprep96 kit (Millipore, Billerica, MA) was used for the isolation of plasmid DNA.

Restriction fragment length polymorphism (RFLP) analysis The PCR reaction was carried out in 96 well plates using the TripleMaster PCR system (Eppendorf, Hamburg, Germany) in a PTC-200 thermocycler (MJ Research Inc., Watertown, MA). Plasmid DNA (200 ng) was digested with $10 \mathrm{U}$ each of BsaRI and $\mathrm{MboII}$ (Fermentas, Hanover, MD) in a final reaction mixture volume of $30 \mu \mathrm{L}$. Gels were stained with ethidium bromide and images obtained using a Kodak photo image system. The clones were then grouped manually based on their RFLP patterns.

\section{High performance liquid chromatography (HPLC) analysis} Extraction and analysis of ergovaline from samples were done according to as described previously (Craig et al. 1994). A Jordi Gel DVB RP 500A HPLC column (Alltech, Deerfield, IL) was used for the analysis. This was attached to an Elmer Perkin series 200 HPLC system. The isocratic mobile phase comprised of acetonitrile/ ammonium carbonate $\left(0.2 \mathrm{mg} \mathrm{mL}^{-1}\right)$ at a ratio of (70:30) run at a flow rate of $1.0 \mathrm{~mL} \mathrm{~min}^{-1}$ attached a flourometer (Elmer Perkin, series 200). The excitation and emission of the flourometer was set at 250 and $420 \mathrm{~nm}$, respectively.

\section{Sequencing and phylogentic tree construction}

Cycle sequencing was performed using the ABI Big Dye Terminator system (Applied Biosystems, Foster City, CA). The SDSC Biology Workbench website (http://workbench.sdsc.edu) was used to manage and analyse sequence data. Partial clone sequences were compared to sequences in published databases using NCBI BLAST and the Biology Workbench which extracted information from the GenBank bacterial sequence database. Only reference sequences of named organisms from the databases with the highest homology scores to the query sequences were chosen for phylogenetic comparison. GenBank reference sequences and clone library sequences 'Align 2' tool found at Oregon State University Center for Gene Research and Bioinformatic. Partial 16S rDNA clone and reference sequences were aligned using the Clustal $\mathrm{W}$ multiple sequence alignment algorithm. Bootstrapping of the alignment was performed 100 times using the ClustalTree.

\section{Results}

Degradation of ergovaline by earthworm gut microbiota

Fig. 1 shows the percentage loss of ergovaline after 7 days incubation with and without earthworm gut contents. In the control (treatment with no earthworm gut slurry added) background loss of ergovaline was $20 \%$ from the initial concentration over the 7 days. Most of this back loss of ergovaline took place on the second day with only slight reduction thereafter. In treatments inoculated with earthworm gut, there was a $60 \%$ decrease in the concentration of ergovaline from the initial concentration of $150 \mathrm{ppb}$ by day 2 . This trend continued and there was further reduction of ergovaline with losses up to $65 \%$.

\section{Earthworm gut clone library analysis}

Fig. 2 represents partial $16 \mathrm{~S}$ sequences and respective operational taxonomic units (OTU's) of the clones represented four major phyla. These phyla are Plantomyces, Chloroflexi, Bacteroides, and Proteobacteria. Clone WGC-A10 showed only $90.2 \%$ similarity to Burkholderia sp. PYX3 ( $\beta$-Proteobacteria). Clone WGC-B01 had $86.1 \%$ similarity to Amaricoccus macauensis ( $\alpha$-Proteobacteria). Clone WGC-B02 had $99.2 \%$ similarity to Algoriphagus dokdonsis (Bacteroides). Clone WGC-B03 had 94.7\% similarity to Uncultured Chloroflexi (Chloroflexi). Clone WGC-B05 had only $91.1 \%$ similarity to Myxobacterium ATI1( $\delta$ - Proteobacteria). Clone WGC-B08 had $93.9 \%$ similarity to Pirellula staleyi (Planctomyces). Clone WGC-B10 had $96.6 \%$ similarity to Ideonella $s p$. B511 ( $\beta$-Proteobacteria). Clone WGC-C03 had $97.0 \%$ similarity to Amaricoccus macauensis ( $\alpha$ Proteobacteria). Clone WGC-C05 had only $91.9 \%$ similarity to Flexibacteriacea bacteria (Bacteroides). Clone WGC-D01 had 96.4\% similarity to ideonella $s p$. B511 ( $\beta$-Proteobacteria).

Figure 1 Decrease of ergovaline concentration over 7 days. Tubes were spiked with $150 \mathrm{ppb}$ of ergovaline and inoculated for 7 days. Samples were drawn out on day 0 , 2, 4, and 7 and ergovaline was extracted. HPLC analysis was performed on the extracted material. represents controls without the addition of earthworm gut. $O$ represents treatments with earthworm gut. All treatments were performed in duplicate.

Decrease in Ergovaline Concentration

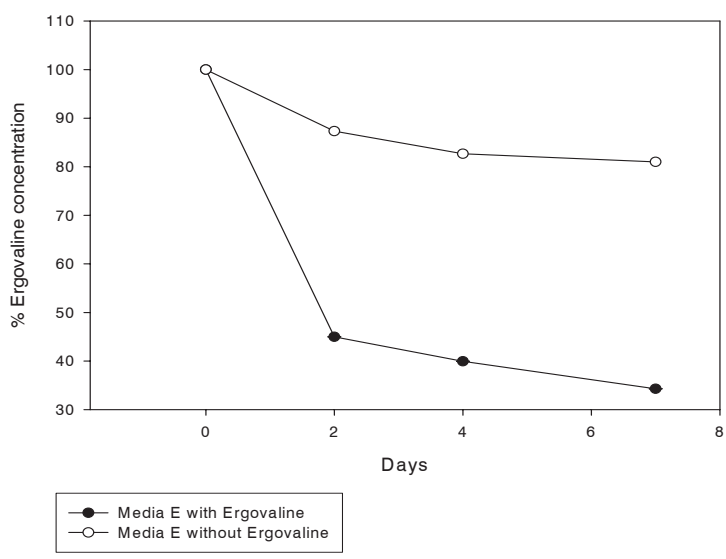




\section{Discussion}

Our novel approach to the ergovaline problem used anaerobic microorganisms obtained from the gut of the "red wiggler" earthworm (Eisenea fetida). There was over $60 \%$ reduction in ergovaline concentration in the worm gut experiments. We did not take epimerization into account due to the inability of the present method to completely resolve the epimer formation as described (Smith \& Shappell 2002). The clonal library was created in an effort to elucidate species that are native to this earthworm involved in detoxifying ergovaline. We obtained only ten OTU's from our clone library construction and analysis. Moreover the number of clones that were picked (144) would not be sufficient for estimating the entire diversity. Though we got PCR products of 1500 base pair, we sequenced only one side of the primer. The 580 nucleotide reading frames we obtained due to this single side sequencing could be reason for obtaining less percentage homology with other bacteria in Genbank. Presently, we are constructing a much more complete clonal library of the earthworm gut flora and sequencing the whole 1500 base pair inserts. Other reasons for such low diversity could also be attributed to factors such as PCR bias in amplification of $16 \mathrm{~S}$ rDNA, extraction of DNA and the selective loss of few species.

This paper provides encouraging preliminary results by identifying the potential use of the earthworm gut flora to detoxify

Figure 2 Phylogenetic analysis of partial 16S sequences from worm gut clones. Neighbor-joining tree of a Clustal W (100 bootstraps performed). Scale bar represents evolutionary distance of 0.02 substitutions per base. Clones are displayed in bold type. Reference sequences are displayed with their GenBank accession numbers.

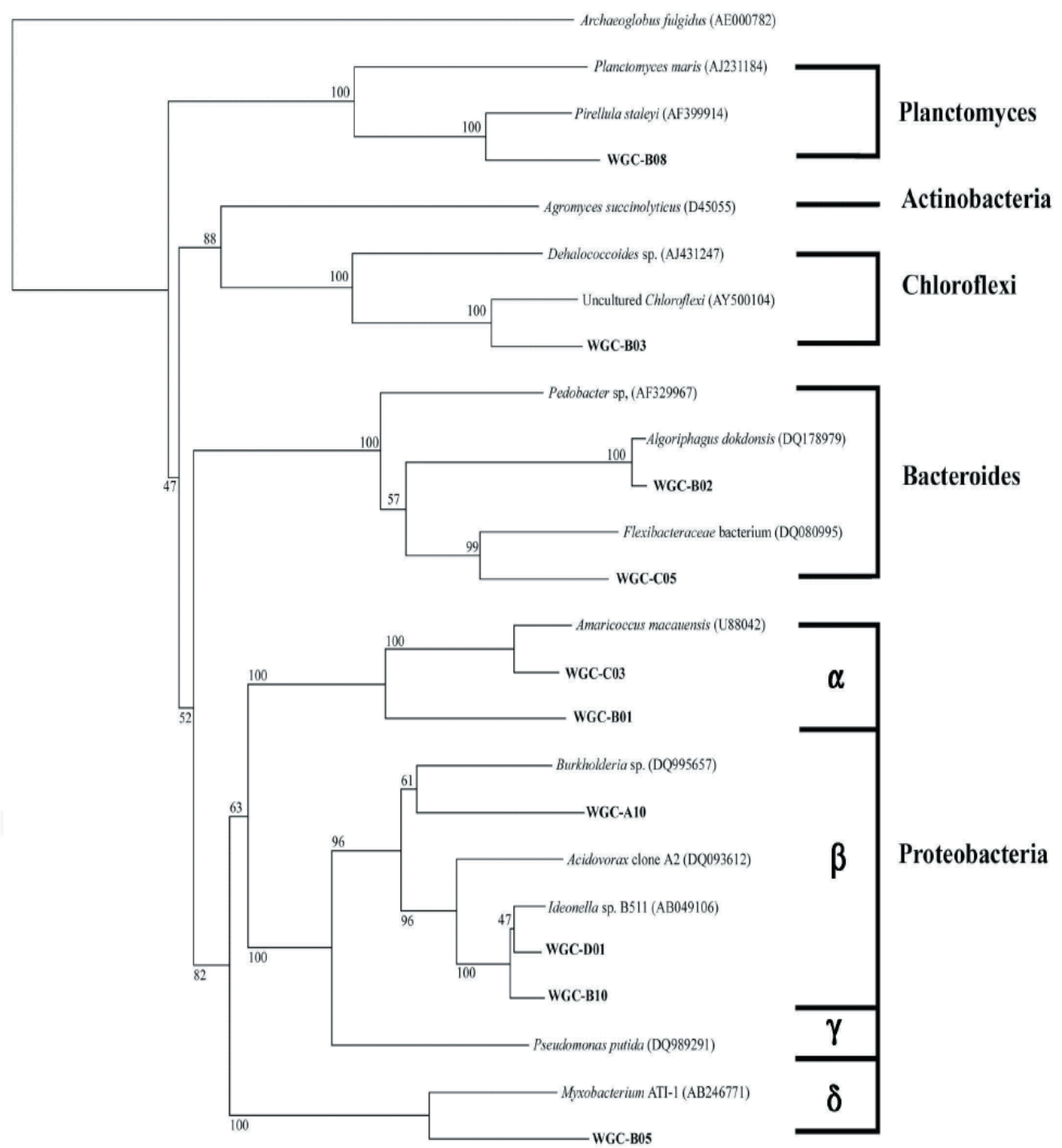


ergovaline. We are also utilising techniques such as LC-MS/MS/ MS for metabolite identification for a better understanding of this complex process.

\section{Conclusions}

There is potential for earthworm gut microorganisms to detoxify ergovaline. The chemistry and molecular biology of this process has not yet been elucidated.

\section{REFERENCES}

Arechavaleta, M.; Bacon, C.W.; Plattner, R.D; Hoveland, C.S; Radcliffe, D.E. 1992. Accumulation of ergopeptide alkaloids in symbiotic tall fescue grown under deficits of soil water and nitrogen fertilizer. Applied and Environmental Microbiology 58: 857-861.

Browning, R. Jr. 2004. Effects of endophyte-infected tall fescue on indicators of thermal status and growth in here ford and senepol steers. Journal of Animal Science 82: 634-643.

Craig, A.M; Bilich, D.K; Hovermale, J.T; Welty, R.E. 1994. Improved extraction and HPLC methods for ergovaline from plant material and rumen fluid. Journal of Veterinary Diagnostic Investigation 6: 348-52.

McDougall, E.I. 1948. Studies on Ruminant Saliva 1. The composition and output of sheep's saliva. Biochemical Journal 43: 99-109.

Porter, J.K.; Thompson, F.N. Jr. 1992. Effects of fescue toxicosis on reproduction in livestock. Journal of Animal Science 70: 1594-1603.

Smith, D.J; Shappell, N.W. 2002. Technical note: Epimerization of ergopeptine alkaloids in organic and aqueous solvents. Journal of Animal Science 80: 1616-22.

Weisburg, W.G; Barns, S.M; Pelletier, D.A. Lane, D.J. 1991. 16S ribosomal DNA amplification for phylogenetic study. Journal of Bacteriology 173: 697-703. 